\title{
Divulgación y enseñanza del patrimonio: interpretación de contenidos digitales y las nuevas perspectivas educativas ${ }^{1}$
}

\author{
Patrimonial education and dissemination: interpretation \\ of digital content and new educational perspectives
}

Cómo citar:

Arango, N. M., Martínez, H. F., \& Pinzón, J. G. (2017). Divulgación y enseñanza del patrimonio: interpretación de contenidos digitales y las nuevas perspectivas educativas. Designia, 5(1), 49-67.

\author{
${ }^{1}$ Este artículo se deriva del proyecto "Apropiación del valor \\ patrimonial tangible a través de las TIC: Ruta Libertadora \\ en Boyacá" desarrollado por el grupo Xisqua; a su vez está \\ enmarcado dentro del macroproyecto "La resignificación \\ patrimonial de la Ruta Libertadora en Boyacá", del programa de \\ Diseño Gráfico de la Universidad de Boyacá \\ *Docente asistente de la Universidad de Boyacá. Investigadora \\ grupo Xisqua. Diseñadora Gráfica de la Universidad de Nariño. \\ Mg. en docencia Universitaria Universidad de Nariño. \\ mnarango@uniboyaca.edu.co \\ https://orcid.org/0000-0002-3357-6511 \\ ** Docente asistente de la Universidad de Boyacá. Investigador \\ grupo Xisqua, Diseñador Gráfico de la Universidad de Boyacá, \\ Mtr. Creación Digital de la Universidad Católica de Valencia España. \\ phermartinez@uniboyaca.edu.co \\ https://orcid.org/0000-0001-5890-7869 \\ ***Docente auxiliar de la Universidad de Boyacá. Investigador \\ grupo Xisqua, Diseñador Gráfico de la Universidad de Boyacá. \\ javgonpinzon@uniboyaca.edu.co \\ orcid.org/0000-0002-4817-8006
}

Palabras clave:

Patrimonio, TIC, contenidos

digitales, educación

\section{Key words:}

Heritage, ICTs, digital contents, education

Recibido: 2/02/2017

Aceptado: 9/06/2017

\section{Resumen:}

Este artículo presenta una revisión bibliográfica referente a la aparición, en particular desde hace dos décadas, de nuevas tecnologías que pueden contribuir a la enseñanza y comprensión del patrimonio cultural. A partir de esto se realiza una clasificación temática orientada a encontrar la relación entre patrimonio, contenidos digitales y educación. Tal revisión 
de antecedentes y casos se propone como estrategia para impulsar el proyecto "Apropiación del valor patrimonial tangible a través de las TIC: Ruta Libertadora en Boyacá", enmarcado dentro del macroproyecto "La resignificación patrimonial de la Ruta Libertadora en Boyacá". Asimismo, como contextualización al objetivo general del proyecto, se propone la generación de herramientas tecnológicas cuyas funciones sean informar, educar y aportar a la apropiación de temáticas, de maneras impensables hasta hace algunas décadas. Enseñar a niños y jóvenes la historia de su país, permitirles revivir y disfrutar la gesta emancipadora, sirve de excusa para la creación de contenidos digitales dinámicos que llamen la atención y vinculen a las nuevas generaciones con el patrimonio nacional, con lo cual también se contribuye a su conservación.

\section{Abstract:}

This paper checks through literature regarding the emergence of new technologies which may support the teaching and understanding of cultural heritage, particularly since the late twentieth century. From this starting point various topics are classified in order to find links between heritage, digital contents and education. Checking antecedents and case studies means an initial strategy for the project "Appropriation of tangible heritage values through ICTs: the Route of Freedom in Boyacá", framed within the major project "Re-signifying heritage in Boyacá Route of Freedom". In addition, technological tools aiming to inform, educate and facilitate the innovative appropriation of topics are proposed as a way to contextualize the project's main objective. Teaching youth their country's history, enabling them to have fun while knowing the Colombian Independence is a key reason for producing dynamic and engaging digital contents which connect new generations with the national heritage. That way, a contribution is also made to such a heritage conservation.

\section{INTRODUCCIÓN}

Según la UNESCO en Banco de la República: 90 años de la banca central en Colombia, el patrimonio es "nuestro legado del pasado, nuestro equipaje en el presente y la herencia que les dejaremos a las futuras generaciones para que ellas puedan aprender, maravillarse y disfrutar de él” (2013, pág. 5). 
En Colombia, dicho patrimonio que identifica al país y lo distingue de los demás, posee tradiciones y elementos heredados de tiempos pasados. Un ejemplo es la "Ruta Libertadora", considerada como Patrimonio Cultural de la Nación, constituida por varios departamentos donde se conservan referentes y vestigios de la gran hazaña de las tropas patriotas que lograron la Independencia de España.

El proyecto al cual se refiere el presente artículo, tiene en el patrimonio delimitado por la especificidad su primera temática, al tratar la Ruta Libertadora en Boyacá, entendida ésta como la extensión geográfica de la gesta libertadora donde se libraron las batallas decisivas. La segunda temática son las Tecnologías de la Información y la Comunicación (TIC), y se delimita por los contenidos digitales y cómo estas herramientas hacen parte de casi todas las actividades de la sociedad contemporánea, al punto que exigen de los ciudadanos la adquisición de nuevas competencias personales, sociales y profesionales para afrontar los continuos cambios en distintos ámbitos. Esto, a su vez, afecta la tercera temática: la educación y las nuevas estrategias de enseñanza y aprendizaje en contextos digitales.

En Colombia, el Ministerio de Tecnologías de la Información ha liderado programas de infraestructura y alfabetización en TIC y acceso a internet. De tal modo, se busca impacto social, generar equidad a través de estas herramientas, fomentar la calidad de la educación bajo un modelo sostenible, permitir a las nuevas generaciones el acceso a infraestructura tecnológica y contenidos digitales que faciliten su formación en espacios tradicionales y no tradicionales, en respuesta al escaso material análogo (libros de texto, guías) en colegios y escuelas rurales y urbanas.

En este orden de ideas, se hace necesario buscar herramientas que permitan al proyecto aquí tratado avanzar en el propósito de generar contenidos que apoyen dichos programas. Además, es indispensable realizar una depuración temática entre patrimonio, contenidos digitales y educación, sin dejar de lado el importante rol cumplido por el sistema educativo actual como un factor clave en la inserción y difusión de tecnologías, temáticas y contenidos en la formación de niños y jóvenes. 
Se empleó una metodología de tipo exploratorio descriptivo para la búsqueda documental y referencial, con el fin de evitar sesgos en la revisión. Mediante el uso de fichas bibliográficas y la selección de documentos pertinentes se propusieron las preguntas y los objetivos. Los criterios y conceptos claves fueron definidos para finalmente depurar y plantear los conceptos a trabajar. A continuación, se presentan los resultados más relevantes de la revisión efectuada.

\section{PATRIMONIO, HISTORIA Y DIVULGACIÓN}

El Ministerio de Cultura, como entidad rectora encargada de formular, coordinar, ejecutar y vigilar la política del Estado colombiano en el sector cultural, clasifica el patrimonio en natural y cultural. Este último es concebido como la suma de bienes y manifestaciones materiales e inmateriales que dan a un grupo humano sentido, identidad y pertenencia. Adicionalmente, lo entiende como factor de bienestar y desarrollo y está consciente de que los colombianos tienen el compromiso y la responsabilidad de velar por su gestión y protección. Hoy en día, el Ministerio apuesta por un enfoque integral para la gestión y salvaguarda del patrimonio cultural. Las políticas públicas en este sentido reconocen a las comunidades el papel fundamental de crear, transformar, heredar y otorgar valor a sus expresiones culturales materiales e inmateriales, pues son finalmente quienes las "usan".

El patrimonio cultural puede ser clasificado en material e inmaterial. El primero, también denominado tangible, corresponde a la materialización de la cultura, lo que podemos tocar. A su vez este patrimonio se subdivide en dos grupos:

- Inmueble: no se puede mover, comprende ciudades, plazas y edificios, entre otros.

- Mueble: conformado por colecciones bibliográficas, documentales, monumentos en espacio público, esculturas, obras de arte, utensilios de uso doméstico.

El patrimonio inmaterial o intangible se entiende como expresiones, conocimientos y prácticas que dan a los grupos humanos un sentido de identidad, pertenencia y continuidad histórica al transmitirse de generación en generación.

El ministerio de cultura de Colombia en la ley 1185 define que el patrimonio cultural está compuesto por:

\footnotetext{
Los productos y las representaciones de la cultura que son expresión de la nacionalidad colombiana, tales como la lengua castellana, las lenguas y dialectos de las comunidades indígenas, negras y creoles, la tradición, el conocimiento ancestral, el paisaje cultural, las costumbres y los hábitos, así como los bienes materiales de naturaleza mueble e inmueble a los que se les atribuye, entre otros, valor audiovisual, fílmico, testimonial, documental, literario, bibliográfico, museológico o antropológico (2008, pág. 1)
} 
Respecto al patrimonio natural, en la publicación Vigías del Patrimonio de la Gobernación de Antioquia, la UNESCO lo definió como:

Los monumentos naturales constituidos por formaciones físicas y biológicas o por grupos de esas formaciones que tengan un valor universal excepcional desde el punto de vista estético o científico, las formaciones geológicas y fisiográficas y las zonas estrictamente delimitadas que constituyan el hábitat de las especies animal y vegetal amenazadas, que tengan un valor universal excepcional desde el punto de vista estético o científico. Los lugares naturales o las zonas naturales estrictamente delimitadas, que tengan un valor universal excepcional desde el punto de vista de la ciencia, de la conservación o de la belleza natural (2011, pág. 34).

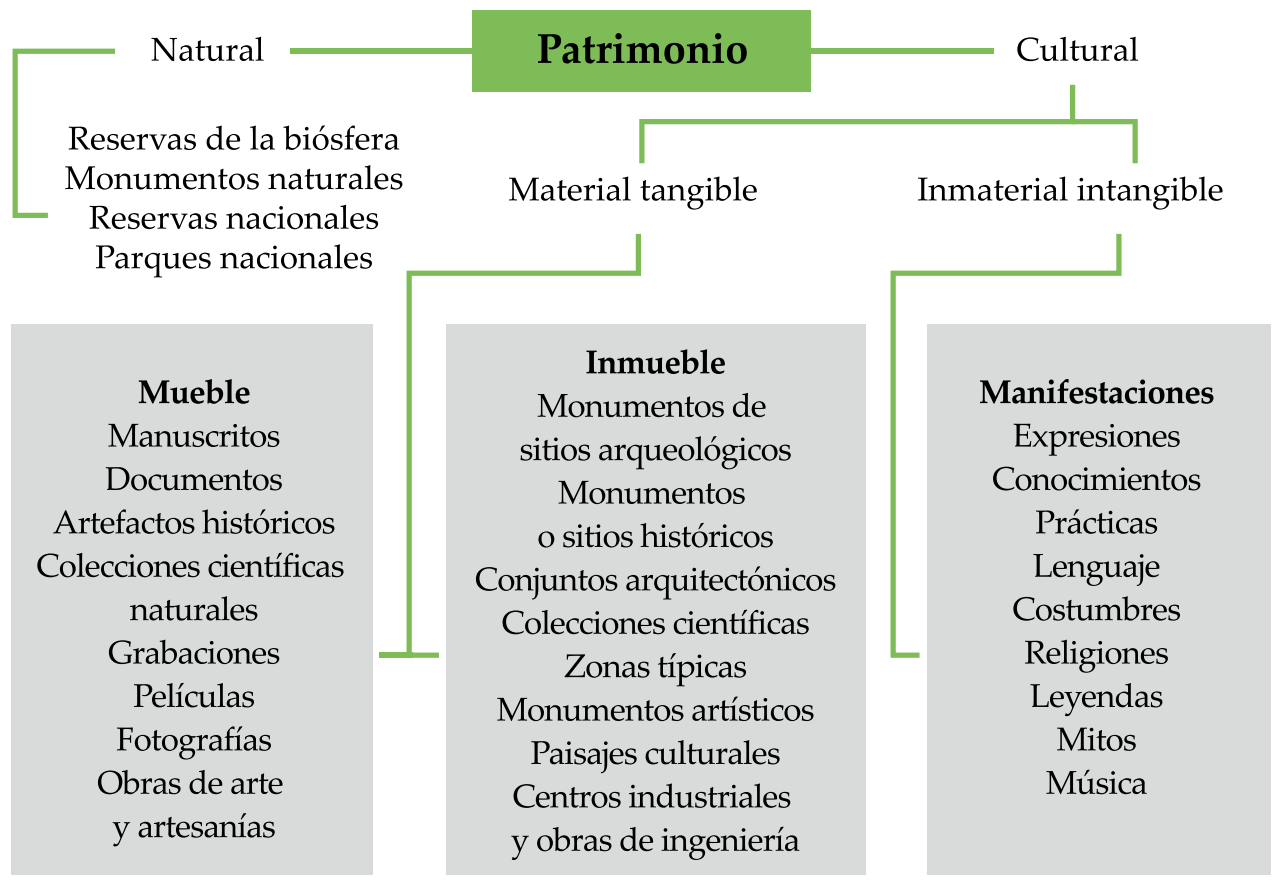

Gráfico 1. Clasificación del patrimonio. 
En el marco de la política pública para la gestión, protección y salvaguarda del patrimonio cultural, el gobierno nacional está interesado en implementar acciones en este sentido a través de estrategias de consolidación. En tal contexto, la investigación aquí expuesta contempla entre sus objetivos la generación de herramientas que aporten a la conservación y apropiación patrimonial. Se ha planteado entonces la elaboración de contenidos digitales educativos orientados a ayudar a niños y jóvenes a apropiar temáticas relacionadas con el patrimonio, lo cual resulta de importancia en la medida que las nuevas generaciones pueden aportar en un futuro al reconocimiento y conservación de los bienes culturales. En el Compendio de políticas culturales realizado por el ministerio de cultura, Juan Luis Mejía dice: "Es importante entender el patrimonio como el derecho a la memoria de las generaciones futuras, que se convierte en deber para las generaciones presentes". (Ministerio de Cultura de Colombia, 2010, pág. 225).

Tradicionalmente, la investigación sobre el patrimonio se ha centrado en análisis disciplinares y académicos (elementos y tipologías patrimoniales, difusión y conservación). Una preocupación actual es cómo el patrimonio pasa por procesos de destrucción paulatina a cuenta del decrecimiento en la sensibilidad hacia su reconocimiento y valoración por parte de las nuevas generaciones. A esto se suma la poca importancia que se da a la tarea de promover estrategias para la alfabetización, difusión y divulgación patrimonial desde las instituciones educativas, como también la inclusión de dicha temática en los contenidos curriculares. Esta es una tarea para los docentes de educación primaria y secundaria.

En el artículo Fundamentos para una enseñanza y difusión del patrimonio desde una perspectiva integradora de las ciencias sociales y experimentales, Estepa, Wamba y Jiménez plantean cuestiones relacionadas con la revitalización del patrimonio:

\begin{abstract}
¿Qué entendemos por este concepto?, ¿qué concepción del patrimonio se transmite desde los museos, exposiciones y en el ámbito escolar?, ¿están dotados los colegios de herramientas adecuadas para leer el patrimonio, comprenderlo, valorarlo, cuidarlo, disfrutarlo y transmitirlo?, ¿qué puede aportar la enseñanza del patrimonio a la enseñanza de las ciencias sociales? (2005, pág. 20).
\end{abstract}

A estos interrogantes cabe añadir el potencial de lo patrimonial como instrumento de alfabetización, para lo cual lo científico-tecnológico ocupa un lugar pues las nuevas tecnologías pueden facilitar el acceso al conocimiento, difusión y divulgación de expresiones culturales, y de tal modo, incrementar su impacto. Se considera entonces pertinente una perspectiva más holística que integre lo educativo, tecno- 
lógico y cultural, para evitar tratamientos disciplinares que reduzcan y dificulten un estudio sistémico y sea posible relacionar cultura con divulgación científica de forma más amplia.

Según Rico Cano (2004), la pérdida progresiva de identidad con respecto al pasado y los elementos patrimoniales ha puesto de relieve la necesidad de la difusión de estos. Además, "la continuidad de esa tradición se ve amenazada en todo el mundo por las fuerzas de la homogeneización cultural" (ICOMOS, 1999, pág. 1). Adicionalmente, Estepa, Aguado y Jiménez (2005) mencionan que en la creciente desvinculación de las personas con su pasado ha influido la falta de acceso a los referentes patrimoniales. Como una respuesta a este fenómeno, Martín (2007) ve precisamente en la divulgación la acción para poner en contacto a los individuos con el patrimonio y permitirles adquirir mecanismos de interpretación para comprenderlo. El objetivo no ha de ser la simple transmisión de información, sino que a través de la difusión se pretende que los destinatarios adquieran actitudes transformadoras de su relación con los bienes patrimoniales, ya que el patrimonio tiene en la educación un medio para su reconocimiento y conservación, la difusión mencionada también debe orientar sus esfuerzos al ámbito escolar. Surge entonces otro horizonte para promover el patrimonio entre las nuevas generaciones y hacerlo sostenible en el tiempo como un legado que les pertenece. Al respecto, resulta válido proponer las TIC como herramientas para tales procesos.

Los medios informáticos y digitales no fueron diseñados específicamente para su uso como recurso educativo, aun así, ofrecen "grandes posibilidades para el trabajo escolar, muchas más, sin duda, que otras tecnologías que se están utilizando en la educación (Martínez Sánchez, 1996, pág. 110).

Sin embargo, es preciso tener en cuenta que estos recursos no son una solución mágica a los problemas y deben insertarse en proyectos estructurados para servir de complemento. 


\section{TIC Y EDUCACIÓN}

Las TIC pueden definirse en dos sentidos: tecnologías tradicionales empleadas en medios comunicativos, principalmente radio, televisión y telefonía convencional, y tecnologías informativas modernas caracterizadas por digitalización de contenidos en la informática, la telemática y las interfaces. Una aproximación no cronológica a las TIC indica un uso de conceptos distintos para hacer referencia a lo mismo.

\section{Todos aquellos medios de comunicación y de tratamiento de la infor- mación que van surgiendo de la unión de los avances propiciados por el desarrollo de la tecnología electrónica y las herramientas conceptuales, tanto conocidas como aquellas otras que vayan siendo desarrolladas como consecuencia de la utilización de estas mismas nuevas tecnologías y del avance del conocimiento (Martínez Sánchez, 1996, pág. 102)}

Cabero (2000) y Carrillo (1997), hacen referencia a la posibilidad de distinguir entre tecnologías convencionales, nuevas tecnologías y tecnologías avanzadas. En el primer grupo incluyen aquellas basadas en el habla, la escritura, el dibujo y la pintura, entre otras. En el segundo, los recursos audiovisuales, la prensa y la televisión. El tercero, por su parte, abarca aquellas relacionadas con el diseño, la animación de software informático, internet y otros campos afines.

De forma semejante, Tirado (1998) distingue entre nuevas tecnologías y tecnologías avanzadas, clasificándolas en relación con la interactividad y la flexibilidad espaciotemporal. A su vez, Majó y Marquès (2002), refieren tres campos de composición de las TIC: informática, telecomunicaciones y tecnologías de imagen y sonido. En una línea similar; Haag, Maeve y McCubbrey, citado en el artículo Las tecnologías de la información y la comunicación (Baelo Álvarez \& Cantón Mayo, 2009), consideran que las tecnologías de información están compuestas por "cualquier herramienta basada en los ordenadores y que la gente utiliza para trabajar con la información, apoyar a la información y procesar las necesidades de información"( s.p.). Para Medina (2007) las TIC se han usado de forma indistinta, centrándose más en el espacio temporal que en las características de los instrumentos, para referir ámbitos de estudio afines. 
En resumen, existen diversas orientaciones para definir las TIC. En su mayoría, hay coincidencia en reconocer la implicación del componente tecnológico y su carácter de herramientas para la comunicación, tal como expresa Cabero (1998):



No obstante, la importancia de estas herramientas para la educación es un horizonte que admite mayor exploración. Frente a esto y con el fin de interpretar el uso de las TIC como recurso para desarrollar una propuesta que intenta articular orientaciones desde la perspectiva educativa con la temática específica del patrimonio, es necesario analizar de qué manera la incorporación de las TIC en los contenidos curriculares contribuye al desarrollo tecnológico y cultural de niños y jóvenes.

Asimismo, las políticas gubernamentales corresponden con dicho propósito al tratarse de una iniciativa que propende la capacitación y formación de docentes y estudiantes en instituciones formativas. En 2009, con la creación del Ministerio de Tecnologías de la Información y las Comunicaciones (Mintic) se buscó promover y masificar la utilización de estas herramientas para fortalecer el sector educativo. A partir de este objetivo surgieron varios proyectos. Uno de ellos es Computadores para Educar, el cual busca generar oportunidades de desarrollo y mejorar la calidad de la educación de niños y jóvenes mediante la dotación de herramientas tecnológicas, la formación y acompañamiento a las comunidades educativas y la gestión ambiental de equipos de cómputo en desuso. Así, desde 2011 lleva estos equipos a colegios en sectores vulnerables en aras de fortalecer su infraestructura tecnológica, fomentar su uso, disminuir la deserción estudiantil y favorecer los procesos de enseñanza de los docentes al capacitarlos en el manejo de los recursos proporcionados. Según la página web del Mintic en el 2016, hasta ese momento habían sido entregados alrededor de 74.000 computadores portátiles en las escuelas públicas del país y se había reducido la deserción en un $4.3 \%$. Vive Digital es otra iniciativa que correlaciona la apropiación de las TIC, la generación de empleo y la reducción de la pobreza. Con este plan se apunta a llevar cobertura tecnológica a lugares remotos para posibilitar en ellos el aprendizaje. Con el apoyo de empresas proveedoras de telefonía e internet se pretende de este modo dar un salto tecnológico mediante la masificación de internet, el desarrollo del ecosistema digital nacional y el uso de la tecnología. 
El informe de gestión presentado por el MINTIC para el periodo junio de 2015 a mayo de 2016 registró un crecimiento en poco tiempo en el cubrimiento de infraestructura y capacitación, lo cual ha beneficiado a gran parte de la población.

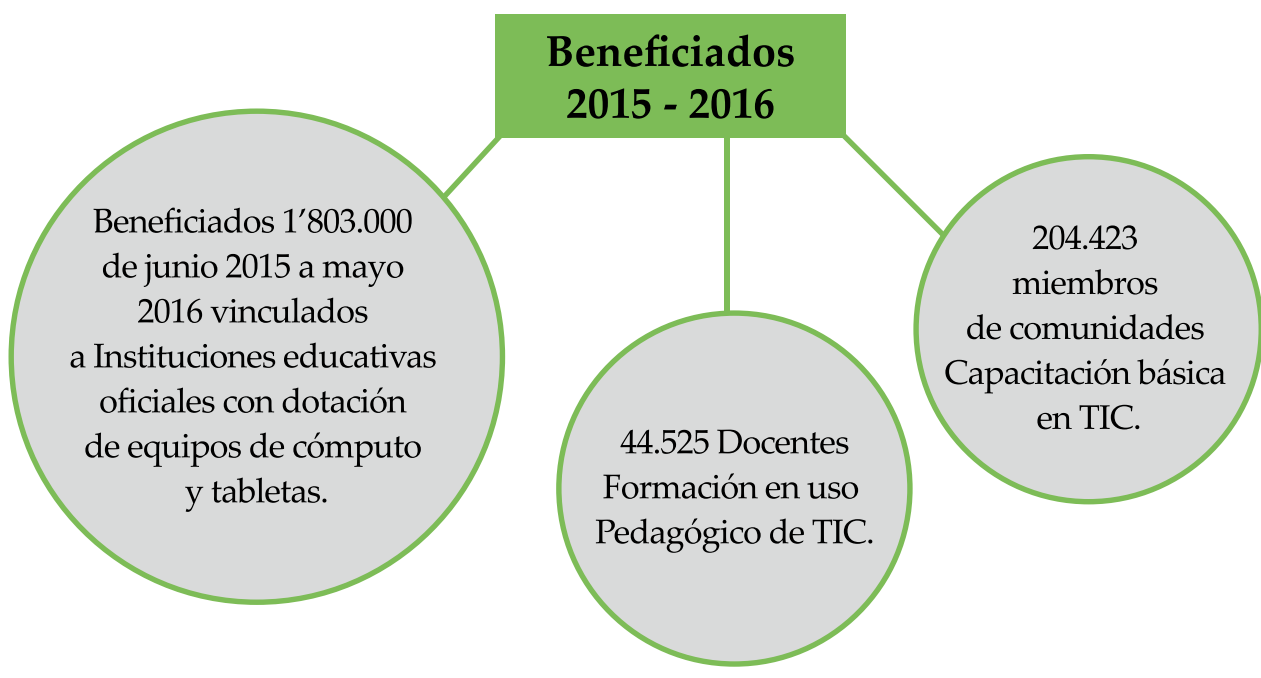

Gráfico 2. Adaptación de beneficiarios del plan Vive Digital para la gente 2015-2016, computadores para educar

Ahora bien, si se desea implementar iniciativas asistidas por las TIC es necesario contar con varios elementos. Gutiérrez y Orozco (2007) mencionan en su artículo Políticas tecnológicas en un escenario de gestión del conocimiento en educación, algunos requerimientos a incorporar en ambientes educativos, como lo son: mejorar las posturas en la retórica tecnocrática y cultura tecnológica, aumentar y mejorar la producción de información, reducir las desigualdades sociales; estas deben ser integradas en los modelos TIC con base a todas aquellas políticas de ciencia y tecnologías establecidas por los órganos gubernamentales. Claro está que, si una escuela "no ha definido su modelo pedagógico y realiza después una integración de herramientas tecnológicas, seguirá produciendo escasos resultados" (Quintanar, 2010, pág. 6). 
Desde esta perspectiva, puede afirmarse enfáticamente que la tecnología por sí misma no mejora el aprendizaje. Es responsabilidad de las instituciones educativas propender el correcto uso de las TIC y las metodologías concomitantes con estas para incentivar a profesores y alumnos a emplearlas. Corresponde al alumno tener disponibilidad para aprender nuevos conocimientos como también al docente adquirir los métodos respectivos y aplicarlos de manera que ambas partes satisfagan las necesidades de aprendizaje y enseñanza.

La educación del siglo XXI es otra. Las TIC han abierto nuevos frentes para la transformación de la enseñanza. Los sistemas educativos contemporáneos se enfrentan a múltiples retos debido a esos cambios y a los paralelos entre los procesos de aprendizaje, la generación de conocimiento y las TIC.

Estas TICs y TACs, han abierto un nuevo panorama ante el que los docentes
y los sistemas educativos deben replantearse nuevos espacios formativos
y por tanto, nuevos contenidos educativos, y por ello unas metodologías
adecuadas que inciden en los procesos de enseñanza-aprendizaje, para
poder garantizar una educación y formación de calidad a los futuros ciu-
dadanos, de manera que sea lo más cercana posible a la realidad de los
nativos digitales (Moya López, 2013, pág. 2).

Sobre el uso de las tecnologías emergentes en la educación y su potencial para transformar contextos tradicionales, según George Veletsianos en (Adell Segura \& Castañeda Quintero, 2012) comenta:

\begin{abstract}
Las tecnologías emergentes son herramientas, conceptos, innovaciones y avances utilizados en diversos contextos educativos al servicio de diversos propósitos relacionados con la educación. Además, propongo que las tecnologías emergentes ("nuevas" y "viejas") son organismos en evolución que experimentan ciclos de sobrexpectación y, al tiempo que son potencialmente disruptivas, todavía no han sido completamente comprendidas ni tampoco suficientemente investigadas (pág. 16).
\end{abstract}

En este panorama se considera pertinente generar e implementar contenidos digitales educativos en las aulas para fortalecer el uso de herramientas tecnológicas y vincular a las nuevas generaciones con emergentes procesos de enseñanza. 


\section{CONTENIDOS DIGITALES Y EDUCACIÓN}

En el ensayo The Pasts and Futures of Digital History, Ayers (1999) afirma que es preciso "entender los nuevos medios y sus posibles implicaciones, tanto por motivos defensivos como por prometedores. Tenemos que resistir a la dilución y la deformación del conocimiento histórico traído por la erosión de nuestra autoridad en un nuevo medio extensamente disperso" ${ }^{\prime 2}$.

En el segundo seminario técnico internacional con la temática, las nuevas tecnologías de la información y comunicaciones (ntics) y el mejoramiento de la calidad de la docencia universitaria, se define el contenido digital como: "Información digitalizada, producida o adquirida con el objetivo de ser accesible e intercambiable para favorecer el diálogo entre sus usuarios". (Gómez Ríos, 2004). Entre sus principales características se encuentran esa capacidad de ser accesibles, como también de ser clasificados, reproducidos y almacenados casi ilimitadamente. Estos contenidos incluyen videos, fotografías, gráficos y música, entre otros. Si bien suelen ser material multimedia digitalizado, aquellos dirigidos a contextos educativos permiten registrar, manejar y comparar información, en dinámicas de trabajo colaborativo propias de los entornos de aprendizaje. La selección y estructuración que de ellos se haga en procesos específicos de enseñanza debe responder a criterios de pertinencia, organización lógica y coherencia con una estrategia pedagógica.

El progreso tecnológico, el desarrollo de los sistemas informáticos y el impacto de estos en la vida cotidiana son factores que han llevado a replantear en cierta medida las metodologías de enseñanza y aprendizaje. La incorporación de los nuevos recursos en las aulas hace indispensable la adquisición de competencias digitales en docentes y alumnos para potenciar procesos acordes a la sociedad del siglo XXI.

${ }^{2}$ Texto originalmente en inglés, traducción de los autores.
(...) los textos digitales en sus diferentes manifestaciones son una realidad y un recurso educativo a disposición de los educadores. Por ello, familiarizarse con los mismos o tener un conocimiento sobre ellos es fundamental para tener nuevas vías para la motivación de los estudiantes en su búsqueda de acceso al conocimiento cultural. (López Valero \& Jerez Martínez, 2015, pág. 179) 
La era digital amplío las posibilidades de los medios tradicionales, que han pasado a coexistir como un complemento. Esta conjunción ha posibilitado actividades interactivas y atractivas, orientadas a estimular el interés de los estudiantes por los contenidos tratados, tanto en el aula como en casa. Los profesores en sus asignaturas suelen ahora usar libros digitales como herramienta alternativa no tradicional. Esto multiplica las posibilidades de los procesos educativos, propicia otras formas de acceder a la información, fortalece el conocimiento y puede mejorar el rendimiento académico. Como expresa García (2012), los contenidos educativos digitales amplían las posibilidades de los libros de texto tradicionales.

Al considerar la incorporación de contenidos digitales en la educación es necesario distinguir los posibles partícipes de los procesos. Prensky (2009) en su versión traducida, identifica como inmigrantes digitales a quienes nacieron antes de la era digital, se han adaptado a la tecnología y hablan su idioma; "la designación que me ha parecido más fiel es la de "Nativos Digitales", puesto que todos han nacido y se han formado utilizando la particular "lengua digital" de juegos por ordenador, vídeo e Internet." Mientras que García, Portillo y Romo (2007) mencionan que: “Estos inmigrantes son fruto de un proceso de migración digital que supone un acercamiento hacia un entorno altamente tecnificado, creado por las TIC". Particularizan que sus edades se encuentran entre los 35 y 55 años y los diferencian de los nativos digitales, nacidos en dicha era y que dentro de 20 años constituirán el $70 \%$ de la población mundial. Entre ambas generaciones las diferencias pueden ser considerables. En contraste con los inmigrantes, con cierta tendencia a guardar en secreto la información (el conocimiento es poder), los nativos la distribuyen con naturalidad, pues creen que es algo para compartir. A su vez, los primeros tienden a actuar de forma reflexiva, por lo tanto, más lenta, en tanto los segundos pueden tomar decisiones con rapidez en ambientes complejos, sin pensarlo mucho.

Es relevante considerar estas realidades y tratar en lo posible que la educación mediada por las nuevas tecnologías no sea excluyente sino, por el contrario, favorecedora de una igualdad que permita el desarrollo personal e integre a las distintas generaciones en torno a procesos emergentes. Por consiguiente, los contenidos digitales deben suplir ciertas consideraciones, como responder de forma dinámica y flexible a la diversidad de estilos de aprendizaje y atender los intereses y las necesidades formativas de quienes participan en dinámicas educativas. 
En consonancia con lo acotado por López y Jerez (2015), estos contenidos deben posibilitar una relación entre educación y cultura como vía de acceso al conocimiento. En tal dirección, Moya (2013) plantea un marco lógico con el cual se espera garantizar su calidad. Dicho formato retoma características tradicionales e introduce aportes desde la perspectiva educativa (gráfico 3).

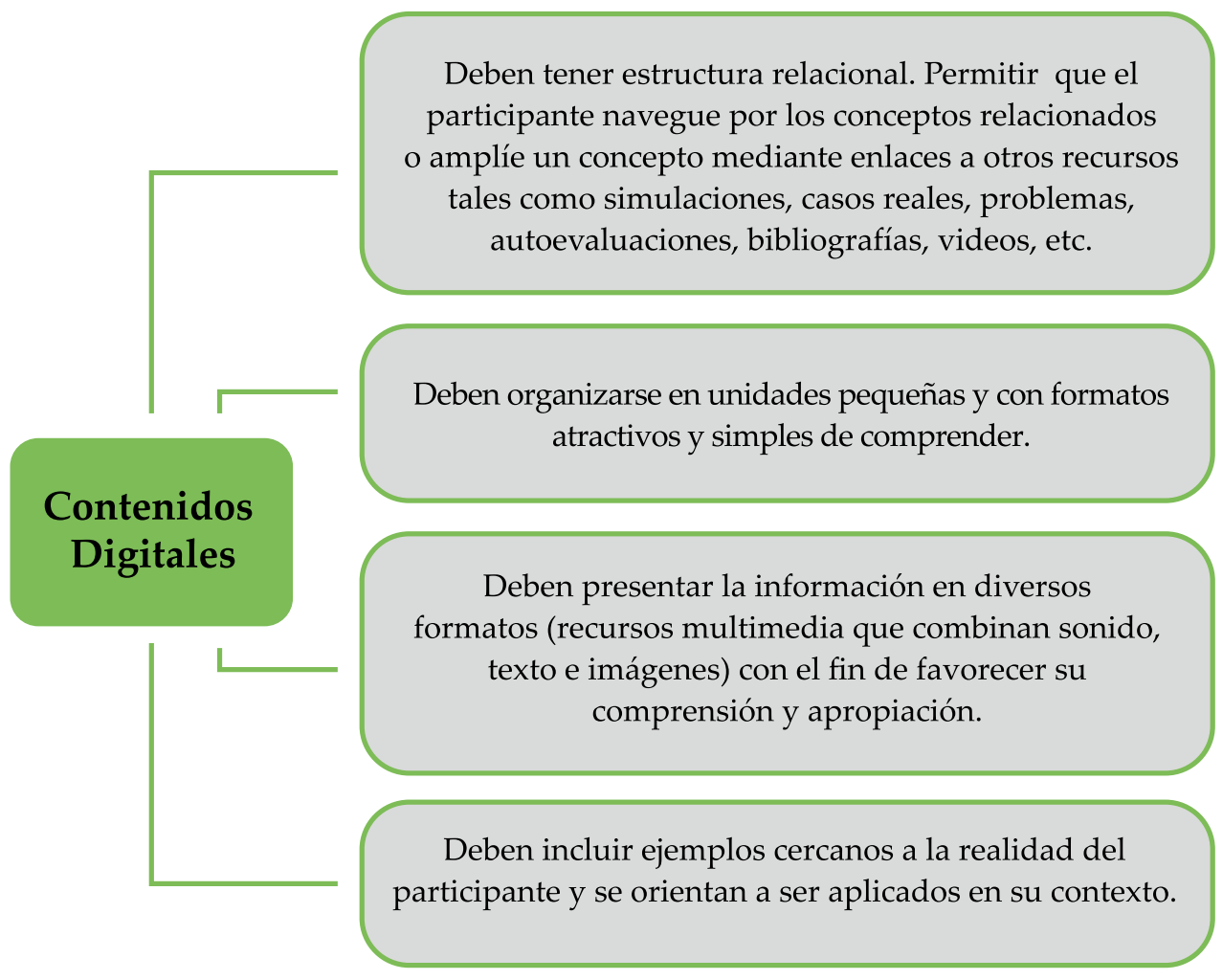

Gráfico 3. Características de los contenidos digitales. Fuente: Moya (2013, pág. 7).

La interacción es otro elemento a tener en cuenta al considerar la producción y el uso de estos contenidos. López y Jerez (2015) mencionan "La persona lectora en la actualidad recibe todo tipo de hipertextos, por lo que es necesario resaltar el concepto de interacción e incidir en la formación del lector que se enfrenta cotidianamente a dichos estímulos" (pág. 172) . La interacción puede comprenderse en dos sentidos: como la establecida entre dos o más personas por medio de la conversación, o bien la definida entre personas y máquinas, por ejemplo, un usuario con un dispositivo electrónico. 
Describe la comunicación entre un usuario/actor y un sistema (informático, video u otro). El grado de interactividad del producto viene definido por la existencia de recursos que permiten que el usuario establezca un proceso de actuación participativa-comunicativa con los materiales. (Estebanell Minguell, 2007, pág. 23).

La interactividad generada por internet ha llevado a una convergencia de medios para la presentación de información. En esta condición multimedia se relacionan e integran diferentes narrativas a través de herramientas y lenguajes que antes actuaban por separado. Al respecto, García (2009) resalta: “El acceso a internet a través del móvil, la descarga de audio y video, las posibilidades de almacenamiento e intercambio de programas por medio de sistemas digitales son fortalezas que modifican el esquema tradicional de los medios de comunicación". (pág. 105). Ahora bien, dicha convergencia "puede entenderse desde espacios muy diferentes: en el ámbito tecnológico, empresarial, en los contenidos, acerca de los usuarios y, por supuesto, de los profesionales de los medios de comunicación" (pág. 105).

El psicólogo educativo Richard Mayer citado por Latapie Venegas (2007) define así la multimedia:

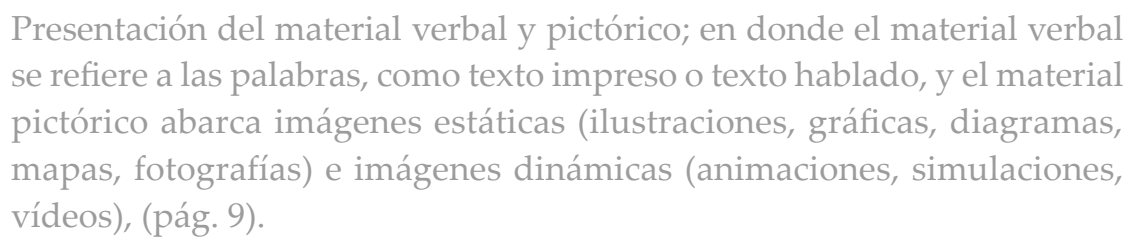

Scolari (2008) conceptualiza diferentes posturas teóricas en torno a los contenidos digitales para reconocer en ellos formas de comunicación que se distancian de las tradicionales. Así, esa convergencia de todo tipo de información digital en un único soporte la refiere como multimedialidad. A su vez, identifica en la digitalización una manera de erradicar la distorsión y pérdida de información; ve en la reticularidad el caso en que el usuario se hace parte del mensaje (por ejemplo, los bloggers) y en la hipertextualidad una vía múltiple de acceso a la información registrada en una red.

\footnotetext{
Si nos centramos en el proceso productivo y en la materia prima de las nuevas formas de comunicación, el concepto clave es la digitalización; si consideramos el contenido (multimedia) y el soporte tecnológico (redes) del proceso de comunicación, la noción que lo distingue es la hipermedia. Si concentramos nuestra mirada en el proceso de recepción de los contenidos la palabra clave es interactividad. (Scolari, 2008, pág. 78)
} 
Para concluir esta revisión teórica, el (gráfico 4) sintetiza conceptos de importancia respecto a la aplicación de contenidos digitales en entornos educativos.

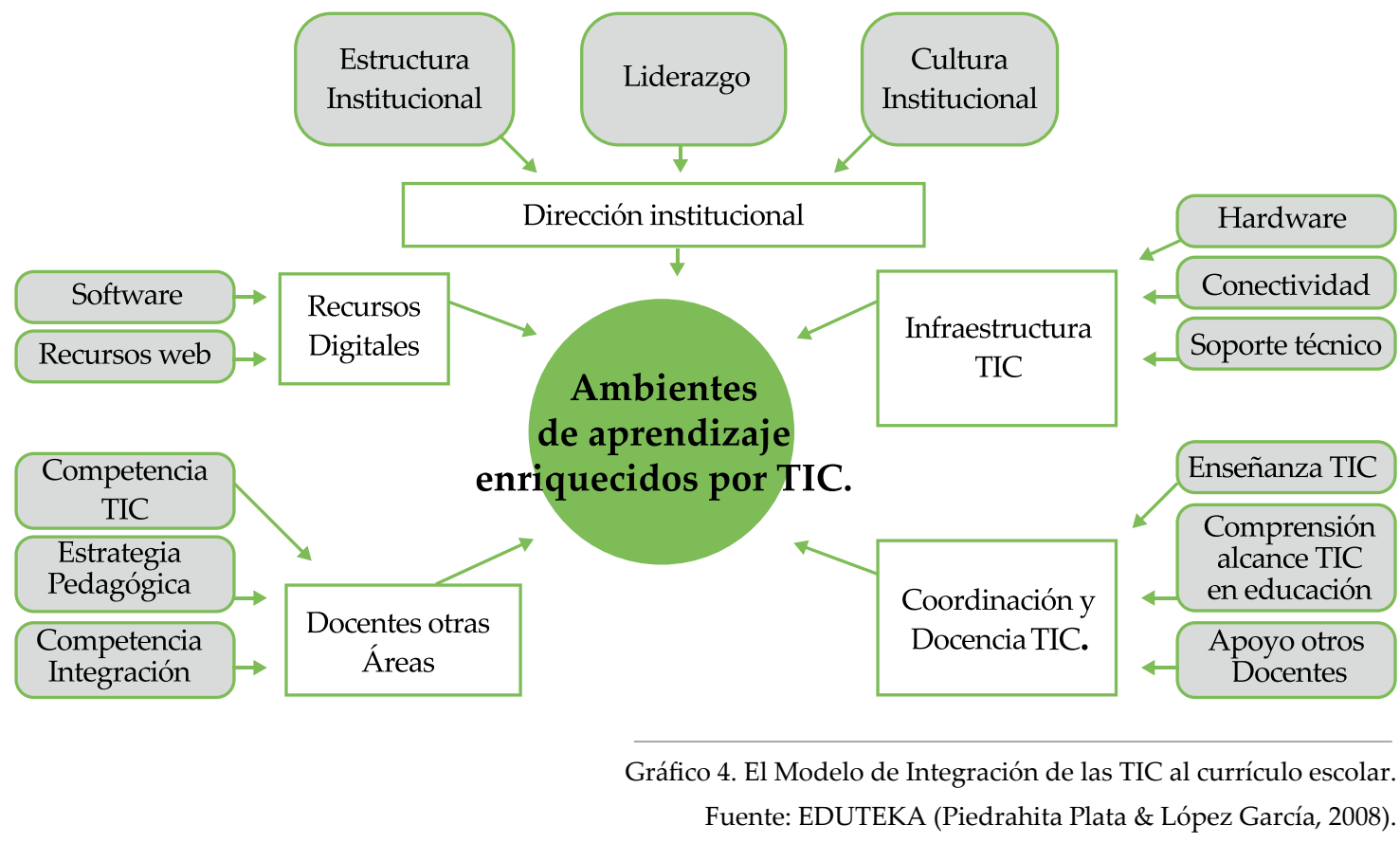

\section{CONCLUSIONES}

La llegada de las TIC a la educación está transformando no solo la forma de enseñar y aprender, sino también las herramientas con que se cuenta para ello. Los contenidos digitales, las nuevas plataformas y los materiales digitales como videowebs y textos interactivos aportan a los emergentes procesos educativos.

Estudios, autores y teorías demuestran las ventajas de emplear contenidos educativos digitales en el aula, dada la flexibilidad que aportan tanto al docente como al estudiante. De igual modo, su incorporación puede fortalecer las competencias digitales de ambas partes. En Colombia, la utilización de tales recursos y herramientas responde a las políticas de alfabetización digital y cubrimiento de infraestructura de internet propuestas desde el gobierno nacional.

Generar contenidos digitales que vinculen las TIC y los medios multimedia, de manera que sean un recurso integrador de temáticas como el patrimonio, significa un reto. El proyecto referido en el presente artículo, consistente en difundir y motivar el reconocimiento y la apropiación de expresiones culturales y marcas de la memoria 
existentes en la Ruta Libertadora de Boyacá, a través de contenidos digitales basados en esos elementos de carácter patrimonial y dirigidos a jóvenes estudiantes, asume justamente ese desafío al destinar las TIC a fines educativos.

Mediante el estudio de los contenidos digitales es posible identificar las características de estos que pueden emplearse para la difusión patrimonial. Dicho trabajo reviste el compromiso de generar herramientas con las que docentes y estudiantes tengan la posibilidad de disfrutar de un aprendizaje más atractivo en ambientes colaborativos. Esto ha sido contemplado en el proyecto aquí referido.

Como afirman Guitert y Corderch (2001) en referencia a la inclusión de tecnologías en la educación, el uso de contenidos digitales y multimedia debe responder a una búsqueda, al compromiso del educador y al momento histórico en que se vive. Estas tecnologías pueden ser un medio eficaz para ensamblar pasado y presente, los tiempos del patrimonio con los cuales se construyen nociones de identidad.

\section{REFERENCIAS BIBLIOGRÁFICAS}

Adell Segura, J., \& Castañeda Quintero, L. J. (2012). Tecnologías emergentes, ¿pedagogías emergentes? En J. Hernández Ortega, M. Pennesi Fruscio, D. Sobrino López, \& A. Vázquez Gutiérrez, Tendencias emergentes en educación TIC (págs. 13-32). Barcelona: Espiral.

Ayers, E. L. (1999). Virginia Center for Digital History Web. Obtenido de http://www.vcdh.virginia.edu/ PastsFutures.html

Baelo Álvarez, R., \& Cantón Mayo, I. (2009). Las tecnologías de la información y la comunicación. Revista Iberoamericana de Educación, 50(7).

Banco de la República. (2013). Banco de la República: 90 años de la banca central en Colombia. Bogotá: Banco de la República.

Cabero, J. (1998). Impacto de las nuevas tecnologías de la información y la comunicación en las organizaciones educativas. En M. Lorenzo Delgado, J. A. Ortega Carrillo, \& T. Sola Martínez, Enfoques en la organización y dirección de instituciones educativas formales y no formales (págs. 197-206). Granada: Grupo editorial Universitario.

Cabero, J. (2000). Las nuevas tecnologías de la información y la comunicación: aportaciones a la enseñanza. En J. Cabero, Nuevas Tecnologías aplicadas a la educación (págs. 15-38). Madrid : Sintesis.

Cano, L. R. (2004). La difusión del patrimonio a través de las nuevas tecnologías. Nuevos entornos para la educación patrimonial histórico-artística. Simposio Internacional de Didáctica de las Ciencias 
Sociales. Formación de la ciudadanía: las TICs y los nuevos problemas. Alicante.

Carrillo, J. A. (1997). Nuevas tecnologías y organización escolar: propuesta eco-comunitaria de estructura y uso de los medios didácticos y las tecnologías. En M. Lorenzo, Organización y dirección de instituciones educativas (págs. 203-222). Granada, España: Grupo Editorial Universitario.

Estebanell Minguell, M. (2007). Interactividad e interacción. Revista Latinoamericana de tecnología educativa RELATEC, 1(1), 23-32. Obtenido de http://relatec.unex.es/article/view/2

Estepa Giménez, J., Wamba Aguado, A. M., \& Jiménez Pérez, R. (2005). Fundamentos para una enseñanza y difusión del patrimonio desde una perspectiva integradora de las ciencias sociales y experimentales. Investigación en la escuela(56), 19-26.

García , F., Portillo, J., Romo, J., \& Benito, M. (2007). IV Simposio pluridisciplinar sobre diseño, evaluación y desarrollo de contenidos educativos reutilizables (SPDECE07). Nativos digitales y modelos de aprendizaje. 3. Bilbao: CEUR Workshop Proceedings.

García Avilés, J. A. (2009). La comunicación ante la convergencia digital: algunas fortalezas y debilidades. Signo y pensamiento, 28(54), 102-113.

García, A. (9 de 11 de 2012). Eroski Consumer, el diario del consumidor. Obtenido de Contenidos educativos digitales, un complemento a los libros de texto: http://www.consumer.es/web/es/educacion/escolar/2012/11/09/214069.php

Gobernación de Antioquia. (2011). Vigías del patrimonbio - Antioquia. Medellín: Secretaría de Educación para la Cultura. Obtenido de http://www.mincultura.gov.co/prensa/noticias/Documents/Patrimonio/ VIG\%C3\%8DAS\%20DEL\%20PATRIMONIO(1).pdf

Gómez Ríos, F. (2004). Segundo seminario técnico internacional las nuevas tecnologías de la información y comunicaciones (ntics) y el mejoramiento de la calidad de la docencia universitaria. Diseño, desarrollo y explotación de contenidos en un entorno internacional, (pág. 52). Monterrey.

Guglielmino, M. M. (2007). La difusión del patrimonio. Actualización y debate. Revista electrónica de patrimonio histórico (1), 195-215.

Guitert i Catasús, M., \& Coderch, J. (2001). ¿Cómo aprender y enseñar con Internet? Cuadernos de pedagogía, 56-63.

Gutiérrez Vargas, G., \& Orozco Cruz, J. (2007). Políticas tecnológicas en un escenario de gestión del conocimiento en educación. Revista iberoamericana de educación (45), 77-88.

ICOMOS. (1999). Carta del patrimonio vernáculo construido. Ratificada por la $12^{\circ}$ Asamblea General de México, Mexico. 
Latapie Venegas, I. (2007). Acercamiento al aprendizaje multimedia. Revista de Investigación de la Universidad Simón Bolívar, 6.

López Valero, A., \& Jerez Martínez, I. (2015). Textualidad digital y multialfabetización. Los contenidos digitales como material educativo. Educatio Siglo XXI, 33(2), 165-181.

Majó, J., \& Marquès, P. (2002). La revolución educativa en la era Internet. (Praxis, Ed.) Barcelona, España.

Martínez Sánchez, F. (1996). La enseñanza ante los nuevos canales de comunicación. En F. J. Tejedor, \& A. García Valcárcel, Perspectivas de las nuevas tecnologías en la educación (págs. 101-108). Madrid: Narcea Ediciones.

Medina, A. C. (2007). Nuevas tecnologías para la educación en la era digital. (Pirámide, Ed.) Madrid.

Ministerio de Cultura - Colombia. (12 de 02 de 2008). MINCULTURA. Recuperado el 17 de 06 de 2016, de http://www.mincultura.gov.co/ministerio/oficinas-y-grupos/oficina\%20asesora\%20de\%20planeacion/Documents/Ley_1185-2008.pdf\#search=1185\%20de\%202008

Ministerio de Cultura de Colombia. (2010). Compendio de Políticas Culturales.

Ministerio de Tecnologías de la Información y las Comunicaciones de Colombia. (2016). MINTIC. Obtenido de Informe de gestión al congreso de la república 2016: http://www.mintic.gov.co/portal/604/ articles-15817_doc_pdf.pdf

Moya López, M. (2013). De las TICs a las TACs: la importancia de crear contenidos educativos digitales. Revista Didáctica, Innovación y Multimedia (27).

Piedrahita Plata, F., \& López García, J. C. (01 de 12 de 2008). Un Modelo para Integrar TIC en el Currículo. Obtenido de EDUTEKA: http://eduteka.icesi.edu.co/articulos/Tema17

Prensky, M. (2009). Marc Prensky. Obtenido de marcprensky.com: https://www.marcprensky.com/writing/ Prensky-NATIVOS\%20E\%20INMIGRANTES\%20DIGITALES\%20(SEK).pdf

Quintanar, A. E. (2010). El impacto de las tic en la educación. UNESCO - Oficina Regional de Educación para América Latina y el Caribe. Brasilia: OREALC.

Scolari, C. (2008). Hipermediaciones: elementos para una teoría de la comunicación digital interactiva. Barcelona: Gedisa.

Tirado Morueta, R. (1998). Las tecnologías avanzadas en la enseñanza: aspectos psicopedagógicos. Comunicar (10), 192-197. 